\title{
INTERACTIVE MULTIMEDIA LEARNING ON HEALTH CARE AMONG LEBANESE WOMEN: AN EXPLORATORY STUDY
}

\author{
Joanna R. Kassem, Lebanese American University, Joanna.kassem@lau.edu.lb \\ Zeinab H. Houssein, Lebanese American University, Zeinab.Housseini@lau.edu.lb \\ Leila Halawi, Bethune Cookman University, drhalawi@gmail.com
}

\begin{abstract}
Multimedia learning greatly enhanced the learning outcome and experience of learners exposed to it. The proposed study will explore the usefulness of multimedia learning software devised to educate Lebanese pregnant women who have a low level of formal education about pregnancy and early infant care. The study will be based on the media richness theory. It is expected that through media rich programs, communication of information is facilitated, promoting the resolving of indecisiveness and ambiguity that stems out of the lack of knowledge or information about a given a subject and the inability to convey a meaning of what is being said respectively.
\end{abstract}

Keywords: learning, multimedia learning, active learning, multimedia instructional message, media richness theory.

\section{INTRODUCTION}

Learning, the cognitive process of acquiring skill or knowledge is facilitated by the incorporation of multimedia tools, such as visual and verbal (auditory) aids $(10,15)$. Multimedia learning is the combination of verbal (words or narration) and non-verbal (diagrams, symbols, and images) representations incorporated to aid learning. (8). Meaningful learning or active learning occurs when the learner gains new information, assembles the information into verbal and visual cues, integrates these representations with previously acquired information, and finally uses the new information in problem solving when presented in a different form (9).

The proposed study will investigate the usefulness of multimedia learning software devised to educate pregnant women who have a low level of formal education about pregnancy and early infant care. The media richness theory, though well known in the organizational and MIS related literature, astoundingly has never been considered in the instructional media design domain. The study will be testing the media richness theory proposed by Daft \& Lengel (5). It will investigate the communicative role that the media richness theory addresses. It is expected that through media rich programs, communication of information is facilitated, promoting the resolving of indecisiveness and ambiguity that stems out of the lack of knowledge or information about a given a subject and the inability to convey a meaning of what is being said respectively (16). Therefore, through the media rich multimedia software, information will be delivered to the participants and meaning will be generated from the delivered information through the organization, structure, and connections prompted by the participants' interaction with the software. Moreover, the study will aim at determining the best arrangement of the components that makes a multimedia software media rich to deliver the information intended. In addition, the multimedia instructional messages will be constructed to suit the individual's personal comprehension capacity. The multimedia instructional messages will be customized to the participants' own need to know the information presented, age, educational level, and culture. To fulfill the previous conditions, the instructional message should be specific, direct and not used excessively so as not to confuse or lose the attention of the participant.

The proposed study will seek to answer the following questions:

1- Will the use of high richness media as opposed to low richness media increase the women's learning score?

2- Will the use of high richness media as opposed to low richness media increase the women's learning satisfaction? 


\section{Issues in Information Systems}

Volume 13, Issue 1, pp. 160-166, 2012

This paper is structured as follows. First, we review the media richness theory from which we infer our research model and hypotheses. We also examine pertinent literature on the topic. We offer in the last section a summary of our current state of research and further research is suggested in concluding remarks.

\section{Media Richness Theory}

Media richness theory was derived from information processing theory, which was developed by Daft, Lengel, and Trevino $(5,4,3)$. They claimed that the communication competence among people is influenced by the fitness of the media and the characteristics of the communication task. Media richness theory spreads the idea that communication richness (or leanness) is an independent property of communication media, and outlines media richness as the capability to expedite common understanding within a time interval. In other words, media richness refers to its ability to enable common meaning and understanding (5).

Daft and Lengel (5) addressed multimedia learning in the light of the media richness theory. The subject discussed by multimedia software should communicate four components (listed below) to be considered media rich. Without these four components the multimedia might attract the learner's attention, however, would be of low educational and learning benefit. First, the multimedia software should prompt a common analysis and understanding of the subject discussed among the learners. This gives the learners common ground of understanding and convergence of viewpoints regarding the subject (16). This component is labeled as the capacity for instant reaction (16). The capacity to transmit multiple cues is another component of the media richness theory. The mixture of animation, video, voice, images, diagrams, numbers rather than focusing on one cue or using text only was necessary to make the multimedia software media rich and thus increased the learning scores of the learners (16). Language variety is very important too. Discussing a certain subject with a multimedia software was best done through the language that the learners were comfortable with. Moreover, each language has its own set of ideas of concepts that are understood by its original speaker and should be addressed carefully (16).

On the other hand, personalizing the media software to suit the needs and preferences of the learners is the fourth component that is crucial for media rich software to achieve its educational purpose. When developing multimedia rich software the medium should have a personal focus. The multimedia software should be devised in a way to suit the sample of learners that is targeted. The gender, orientation, age group, ethnicity, educational level, and culture of the learners should be determined prior to creating the multimedia software program so that it would be appropriate for the receiver (16). Thus, choosing the right design through incorporating the four components of a media rich program discussed to ensure the optimal transfer of information to the participants is necessary (16).

\section{EMPIRICAL STUDIES}

A multimedia instructional message is a presentation consisting of words and pictures that is designed to facilitate meaningful learning (9). The words can include printed or spoken text and the pictures can include static graphics (such as illustrations, maps, charts, and photos) and dynamic graphics (such as animation and video). Pastore (14) examined the effect of audio speed in multimedia instructional messages on multimedia learning. Short, moderate, or long instructions narrated to the students at different speeds affect the students learning process. Moderately compressed instructional messages that were not too short or long aid the learning process best (14). The students would understand what is being said and integrated it in their memory. Long instructional messages contributed in the loss of attention and interest in what is being said. Too short instructional messages, however, were not enough for the student to clarify the meaning of the instructional message (14). Thus, having a regular or somewhat compacted instructional message does not confuse the students and promotes learning.

Mayer (8) tested four instructional design methods and their effects on multimedia learning with a sample of students. The multimedia effect, the coherence effect, the spatial contiguity effect, and the personalization effect were the design methods investigated. These methods work across different media environments (books and computers) (9). The results were as follows: 


\section{Issues in Information Systems}

Volume 13, Issue 1, pp. 160-166, 2012

Multimedia effect. Meaningful learning substantially increased when text is presented with visual aid (pictures or diagrams) or narration (9).

Coherence effect. Adding extraneous information that is unnecessary impaired meaningful learning. Including such irrelevant details made students pay attention to the unimportant information thus impairing the learning process. Excluding such details improved significantly the acquiring of knowledge (9).

Contiguity effect. Contiguity effect stressed that learning is facilitated when the distance between word and pictures in the multimedia instructional message was nearer rather than further from each other on the page or screen. The proximity of wording and pictures allowed their presence simultaneously in working memory, which enhanced the process of integrating visual and verbal models, a key step in active learning (9).

Personalization effect. Meaningful learning was enhanced when the wording presented was in an informal style with a personalized or customized feeling to it (9).

In an experiment by Butcher (2) diagrams were used to test their effects on learning regarding the circulatory system. Students were assigned randomly to a group exposed to text, text with a simplified diagram, or a text with a detailed representation. The incorporation of diagrams significantly improved the students' mental models whereas those exposed to text only showed the least improvement. This further supported the hypothesis that states that acquiring concepts was enhanced upon the incorporation of diagrams (2). Profound conception and memory retention prevailed when text was combined with a multimedia presentation as opposed to text only (2). Factual learning, learning key concepts and facts, was increased through the use of a simplified diagram and not a more complex one (2). Highlighting the essential relationships that were more evident in a simplified diagram promoted the generation of important inferences during learning. On the other hand, the presentation of both visual and verbal materials induced better learning in students, and was mainly accounted for because of repetition. Visual material would add redundancy and therefore would result in better comprehension (1).

In a research conducted by Fiore, Haydee, and Randel (6) diagram presentations were tested to determine how they affected different types of knowledge acquisition. College students were exposed to a diagram presentation as opposed to a controlled sample that was asked to draw information from text only. When exposed to diagrams, participants' integrative knowledge (how to do a certain task or concept function) was affected and not the declarative knowledge (what a certain task or concept is about) (6). Moreover, they found that diagrams offered a scaffold to the formation of more complex mental models. Diagrams helped in establishing interconnections between concepts that appear distant and unrelated when represented through text. Not only the number of interconnections the diagram provided was important, but also the nature of these interconnections facilitated the formation of a more complex mental model. Problem solving in novel non-routine problems relies on the establishment of such complex models.

Pantziara, Gagatsis, and Elis (13) conducted an experiment to test the effectiveness of visual representation on problem solving. They found that visual representations were necessary factors for efficient problem solving, however, they added that such techniques have their limitations. The presence of visual aids such as diagrams did not increase the overall performance of students and rather confused students when solving non-routine problems. Students failed to determine the structure of the problem by viewing the diagrams. When used correctly, students reported that diagrams reduced the difficulty of the problems by guiding the students to the correct solution. Thus, Pantziara et al. (13) concluded that the incorporation of diagrams helped some students in non-routine math problem solving while it impaired solving for others. This variation in results was attributed to different reasons such as students' failure to understand the diagrams or their inexperience in solving math problems in the presence of diagrams. Some students did not have the acquired skill to solve problems with the help of diagrams. They were not able to infer the solution hinted upon by the diagram. For those who were able to understand diagrams, students chose one strategy when several strategies might apply. A diagram would direct a student's thinking one way and not the other. Therefore the student would establish "representational determinism" in which the representation 


\section{Issues in Information Systems}

Volume 13, Issue 1, pp. 160-166, 2012

would help elect one solving strategy and eliminate the use of other strategies (13). This determinism might constrain thinking preventing creativity in problem solving.

Electronic learning is a type of multimedia learning where knowledge is attained through the use of computerized systems and the Internet so that students can have easy access to the content anywhere anytime (12). In a study by Ortiz et al (12), video learning was compared to regular text book learning in a sample of molecular biology students. Molecular biology lectures were recorded and formatted in a way to capture the professor teaching the course and the display of the power points at the same time. These lectures were displayed online and formatted to suit computers, mobile phones, MP4 players and i-Pod video. Therefore, this facilitated easy access of the recorded material anywhere, anytime. Ninety five percent of the students that participated in the research used the videos to advance their learning and reported benefits from the posted material. Moreover, ninety five percent of the students reported that the easy accessibility through any device anywhere allowed for greater participation, interest, and engagement in the biology courses.

Susskind (17) conducted a research to test the effect of learning through power point presentations. Participants were divided into two groups. The experimental group received lectures in power point while the control group received regular lecturing style with no visual aids. Power point presentations enhanced students' attitudes toward the general lectures that they were presented, however, there was no reported difference between regular lecturing and the use of power point on the learning performance of the students. Learning scores were practically similar for both. Moreover, presentations did not affect students' studying patterns. Power point presentation, however, had many advantages. Presentations motivated and encouraged students to take notes in class (17). The structure and organization offered by these power point presentations promoted a clearer understanding of the subjects discussed. The bullet format used enhanced student's feelings that they are encompassing all the major points in the lecture without missing out on any (17). The formal aspect of PowerPoint presentations reduced questions and class interruptions with decreased spontaneity in lectures. This decrease in spontaneity frustrated some students due to the lack of discussion.

Nielsen et al. (11) wanted to test the efficacy of a multimedia software-learning program, The Daktari, which would be used to teach sixty-two livestock keepers in Nairobi about livestock diseases. The program was devised to teach the keepers about three diseases that infect livestock: liver fluke, mastitis, and mange. The software was comprised of visual and auditory aids of a vet, the Daktari (in Kiswahili) and a social worker, Jane, that when selected explain the diagnosis, symptoms, cure, and prevention of the three diseases. The visual-auditory aids were an advantage for the illiterate sample. After exposing the livestock keepers to the program, learning increased regardless of the prior knowledge that these keepers had for the disease. They found that the learning uptake was higher in the older age groups than that of the younger age groups. Although the youngest group had more years of formal education (five or more for all participants under 25 years of age) and scored higher on the prior knowledge pre-tests regarding the three livestock diseases, their knowledge uptake was lower than the older age groups who are much less educated.

Sun and Cheng (16) tested the effectiveness of a media rich software that contains the four components discussed in the theory section on the learning capability of a sample of high school students. A Chinese poem "The Merchant's Wife" is a poem from the Chinese heritage that is known for its complexity among the Chinese students because of the abstract concepts that it contains. The software included animation, images, and music that manifested the poem's components. As a control mechanism, a software containing text only was administered to the control group. Learning scores were significantly higher for the group exposed to the rich media software as opposed to the controlled one, receiving the low media software presenting text only.

\section{RESEARCH MODEL \& METHODOLOGY}

In our proposed study we would be testing the learning capability of Lebanese woman regarding pregnancy in a similar manner to that of Sun and Cheng's (16) research. We propose a research model and two major hypotheses 


\section{Issues in Information Systems}

Volume 13, Issue 1, pp. 160-166, 2012

relative to the design of multimedia instructional material for women with low formal education as shown in Figure. 1. In our research model, learning performance is measured by an objective measure (score) and a subjective measure (learning satisfaction).

The proposed study will be investigating two hypotheses:

H1: The use of high richness media to deliver information to the participants will increase their learning score in comparison to the use of low richness media like text from a book.

H2: The high richness media will increase the participants' learning satisfaction as opposed to the use of low richness media.

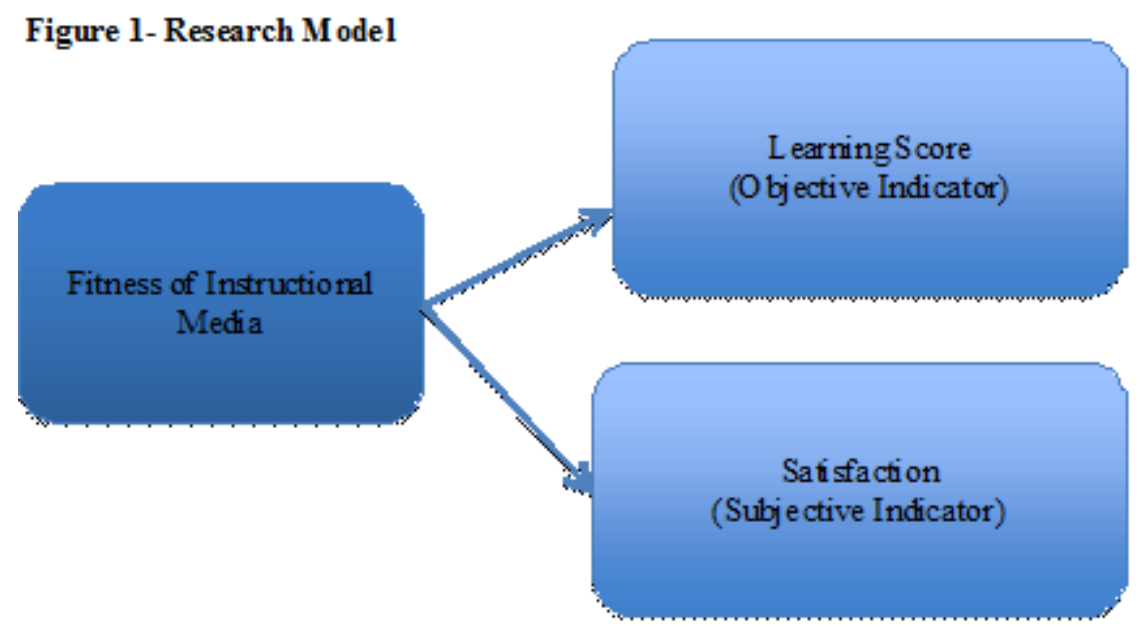

\section{Participants}

A multi stage stratified random cluster sampling of pregnant Lebanese women with school education below grade 12 and a low socioeconomic status will be selected from different areas of Lebanon.

\section{Instruments}

Computer. A touch screen computer will be used. The participants' are not necessary computer-literate and might not have previous experience with keyboards. Thus, the incorporation of touch screens will facilitate the switch between topics without the aid or interaction with outside help limiting the experimenter's expectancy and single operations effect thus enduring construct validity. Therefore we would be using an I pad as the main tool in this experiment. Moreover, all input information will be analyzed using SPSS and will remain anonymous.

Pretest and posttest. The participants will undergo a pretest prior to the experiment to determine the level of knowledge they have about pregnancy. The same test will be administered after exposing the participants in the experimental and control group to the multimedia software as a posttest. The test is comprised of multiple-choice questions concerning the material viewed. Testing can be a threat to internal validity. The presence of a control group, however, will rule out the effects of testing since this group undergoes repeated testing without the intervention. (7) Pretest sensitization may occur threatening external validity. Its effect is discussed in research design (7).

Learning satisfaction questionnaire. To assess their learning experience and satisfaction with the process, participants will fill a learning satisfaction questionnaire. Measurements are analogous to a seven point Likert Scale with one, which stands for strongly disagree all the way to seven, which stands for strongly agree. Five questions 


\section{Issues in Information Systems}

Volume 13, Issue 1, pp. 160-166, 2012

would be addressed to the participants analogous to that provided by Sun \& Cheng (16).The instrument is highly reliable and valid across different populations according to tests conducted by Sun and Cheng (16).

\section{Research Design}

The participants will be randomly assigned in a pretest-posttest control group design. The experimental study will measure the level of change obtained (amount of knowledge gained after exposure to the software) as a function of the intervention (the rich media multimedia software) making the pretest-post test control group design ideal. The experimental group will be exposed to a rich media multimedia software while the control group will be exposed to a low media multimedia software offering a text only module. A pretest and a posttest will be administered before and after the experiment to assess knowledge. Administration of a pretest will aid in matching knowledge levels between participants and then conducting a random assignment to ensure internal validity (7). Moreover, it will enhance the statistical analysis of the results obtained ensuring statistical conclusion validity. The intervention is administered for a short period of time (20 to $40 \mathrm{~min}$ ) between the pretest and the posttest for both groups ruling out internal validity threats such as history, maturation, testing, and instrumentation (7). The pretest might pose a threat to external validity. When participants are affected by pretest sensitization effect, their answers on the posttest are influenced by the answers in the pretest and thus the findings generated cannot be generalized to populations other than that who received the test (7). In the case of the proposed study, pretest would not have much influence on the findings since administration of the intervention is expected to introduce drastic change in the amount of knowledge these women have. Such results cannot be attributed to pretest sensitization alone. In the case of attrition, which is inevitable if it will happen, this design will provide us with information regarding the knowledge level of the participants that dropped out and how the results would have been affected (7). Since all information will be directly loaded to a computer and analyzed in the process, the experimental design is basically a "stand-alone" model that limits the researcher's interaction with participants. Therefore, through this design construct validity is ensured.

\section{Experimental Procedure}

Multimedia Software. A computer programmer will devise the multimedia software containing topics relevant to pregnancy and early infant care. The media rich version of the multimedia software is the intervention. Administering the media low version of the multimedia software will be considered the no intervention condition. For the media rich version, the information supplied by the" Patient Education Pamphlet" provided by the American Congress of Obstetricians and Gynecologists will be summarized in animation and sounds with the presence of diagrams and pictures. However in the low media version, the pamphlet will be presented in plain text. Since the sample used is of Arabic origin, the language used in the multimedia software will be generated in Arabic. Translation validity from English (the language used in the pamphlet) to Arabic will be ensured using a certified translator.

Collection of Participants. Visits will be conducted to different gynecologists in Beirut, Beqaa, South, and North of Lebanon in search for female participants that fit the participants' criteria. Primarily we are looking for participants with a low level of formal education, pregnant or recently married, aged between 18 and 35 . Therefore, participants will be enrolled through the gynecologists visited. An informed consent will be provided.

Procedure. A brief introduction about the software, its content, and touch-screen protocol is given. Participants will use a touch-screen computer to conduct the pretest, interact with the multimedia software, conduct the posttest and fill the learning satisfaction questionnaire. Headphones will be provided to insulate the participants from their surrounding environments. The experiment will be done on an individual basis at a local facility that is close to the area where the participant resides since it would be inconvenient for a pregnant woman from Beqaa to participate in the experiment in Beirut. These facilities such as hospitals, clinics, or public schools will be determined later. 


\section{Issues in Information Systems}

Volume 13, Issue 1, pp. 160-166, 2012

\section{CONCLUSION}

This is a research in progress, we expect to complete the translation of the testing instrument within a month. We did start contacting local hospitals, clinics and public schools in the area identified. Several benefits can be drawn from this study. Our results should provide useful guidelines and framework for educators and multimedia courseware engineers to evaluate the suitable level of media richness required for the course items under consideration. Second, based on findings from this research, we believe that developing suitable instruments for different learning groups such as low income and underprivileged people, elementary students, university students is a worthwhile extension of this research.

This study is among the first in Lebanon to apply and test media richness theory regarding the effectiveness of multimedia instructional material design.

\section{REFERENCES}

1. Bucciarelli (2007) How the construction of mental models improves learning. Mind and Society 6, 67-89. doi: 10.1007/s11299-006-0026-y

2. Butcher K. (2006) Learning from text with diagrams: Promoting mental model development and Inference generation. Journal of Educational Psychology 98, 182-197. doi:10.1037/0022-0663.98.1.82.

3. Daft, R. L., Lengel, R. H., \& Trevino, L. K. (1987). Message equivocality, media selection, and manager performance: Implications for information systems. MIS Quarterly, 11(3), 355-366.

4. Daft, R. L., \& Lengel, R. H. (1986). Organizational information requirements, media richness and structural design. Management Science, 32(5), 554-571.

5. Daft, R. L., \& Lengel, R. H. (1984). Information Richness: A new approach to managerial behavior and organization design. In B staw, \& L.L. cummiges (Eds), Research in Organizational Behavior, 6, 191-233.

6. Fiore S.N., Cuevas, H.M. \& Oses, R.L. (2003). A picture is worth a thousand connections: The facilitative effects of diagrams on mental development and task performance. Computers in Human Behavior, 19(2), 185-199.

7. Kazdin, A.E. (2003). Research design in clinical psychology. Boston, MA: Pearson.

8. Mayer, R. E. (2005). Introduction to multimedia learning. In R. Mayer (Ed.), The Cambridge handbook of multimedia (pp. 135-146). NY: Cambridge University Press.

9. Mayer E., (2003) The promise of multimedia learning: Using the same instructional design methods across different media. Learning and Instruction 13, 125-139. doi: 10.1016/S0959-4752(02)00016-6

10. Mayer, R. E. (2001). Multimedia learning. New York: Cambridge University Press.

11. Nielsen L., Heffernan C., Lin Y., Yu J., (2010) The Daktari: An interactive, multi-media tool for knowledge transfer among poor livestock keepers in Kenya. Computers \& Education, 54, 1241-1247. doi: 10.1016/j.compedu.2009.11.010

12. Ortiz, M., Carvajala, D., Corala, A., Barriosa, P., Henao, C., (2010) Implementation of podcast and clickers in two biology courses at Los Andes University and impact evaluation in the teaching- learning process. Procedia Social and Behavioral Sciences, 2, 1767-1770. doi:10.1016/j.sbspro.2010.03.981

13. Pantziara M., Gagatsis A., Elia H. (2009) Using diagrams as tools for the solution of non-routine mathematical problems. Educational Studies in Mathematics 72, 39-60. doi: 10.1007/s10649-009-9181-5

14. Pastore, R. (2010) The effects of diagrams and time-compressed instruction on learning and learners' perceptions of cognitive load. Educational Communications and Technology 58, 485-505. doi: 10.1007/s11423-009-9145-6

15. Schnotz, W., \& Lowe, R. (2003). External and internal representations in multimedia learning. Learning and Instruction, 13, 117-123.

16. Sun P. \&Cheng H. (2007). The design of instructional multimedia in e-Learning: A Media Richness Theory-based approach. Computers \& Education, 49, 662-676. doi: 10.1016/j.compedu.2005.11.016

17. Susskind (2005) PowerPoint's power in the classroom: Enhancing students' self-efficacy and attitudes. Computers \& Education, 45, 203-215doi:10.1016/j.compedu.2004.07.005. 